\title{
PIGMENT PRODUCTION IN BACTEROIDES
}

\author{
OONAGH Tracy \\ Department of Clinical Microbiology, Royal Infirmary, Glasgow
}

ONE member of the genus Bacteroides-Bacteroides melaninogenicus-characteristically produces a jet-black pigment when grown on blood agar. This property was described by Oliver and Wherry (1921) in the original account of the organism, and has since been regarded as highly distinctive. The work described in this paper is based on the chance observation that other members of the genus are also capable of pigment production.

Originally the black pigment of $B$. melaninogenicus was believed to be melanin (Oliver and Wherry). Subsequently the nature of the pigment was investigated by Schwabacher, Lucas and Rimington (1947). They identified it as haemin, but their cultures were grown on blood agar. Since pigment production by other members of the genus has now been recognised, a study of the pigment produced by these bacteria as well as that of $B$. melaninogenicus seemed to be of interest.

\section{MATERIALS AND METHODS}

Throughout this study, cellular morphology was checked routinely by Gram staining, and after incubation the purity of the cultures was tested by subculture on blood agar. Before the investigation was commenced the fermentation reactions of the strains employed were studied, using the medium of Reed and Orr (1941).

Organisms. The following strains were obtained from the National Collection of Type Cultures: Bacteroides melaninogenicus (NCTC9336), B. fragilis (NCTC9343) and B. necrophorus (NCTC7155).

Chemicals. Haemin (British Drug Houses Ltd, Poole, England) was prepared aseptically as a 0.1 per cent. $(\mathrm{w} / \mathrm{v})$ solution and stored in the refrigerator at $4^{\circ} \mathrm{C}$. The vitamin $\mathrm{K}_{1}$ source used was "Konakion 10" Phytomenadione with polyethylene glycol ester as a dispersing agent (Roche Products Ltd, Welwyn Garden City, England). It was supplied sterile.

Media. Stock cultures were maintained by weekly transfer on 5 per cent. (v/v) horse blood agar, and grown in an anaerobic jar of modified McIntosh and Fildes pattern (Baird and Tatlock, London, Ltd).

Both undefined and partially defined media were used for pigmentation studies. The undefined media were Robertson's cooked meat medium (Cowan and Steel, 1965, modified from Lepper and Martin, 1929) and modified Starkey's medium (Postgate, 1959).

Partially defined media were supplemented with sterile ox serum $4 \mathrm{ml}$ per cent. (v/v) and haemin 0.001 per cent. $(\mathrm{w} / \mathrm{v})$ for the cultivation of $B$. melaninogenicus. Sterilisation was by autoclaving at $15 \mathrm{lb}$. per sq. in. $\left(121^{\circ} \mathrm{C}\right)$ for $20 \mathrm{~min}$., unless otherwise stated. The serum was sterilised by Seitz filtration.

The composition of the partially defined medium is shown in table I.

Solution A was first prepared and dispensed in 100-ml quantities. After sterilisation it could be stored for a maximum period of $1 \mathrm{mth}$. The ingredients of solution $\mathbf{B}$ were dissolved in the solvents recommended by Meynell and Meynell (1965), with aseptic precautions. The concentration of the biotin solution was $0 \cdot 1 \mu \mathrm{g}$ per $\mathrm{ml}$ and of the cyanocobalamin 
$1 \mu \mathrm{g}$ per $\mathrm{ml}$. All the other constituents were prepared as $1 \mathrm{mg}$ per $\mathrm{ml}$ solutions. These vitamin stock solutions were stored at $4^{\circ} \mathrm{C}$ for a maximum of $1 \mathrm{mth}$. Solution $\mathrm{B}$ was prepared by adding the vitamins in the volumes stated and the volume was brought to $100 \mathrm{ml}$ by adding the requisite amount of solution $A$. The solutions of glucose $(20 \mathrm{~g}$ per cent. $\mathrm{w} / \mathrm{v}$ in distilled water) and of ferrous sulphate $\left(\mathrm{FeSO}_{4} \cdot 7 \mathrm{H}_{2} \mathrm{O}\right.$ in distilled water 5 per cent. w/v) were prepared separately and sterilised by autoclaving.

\section{TABLE I}

Constituents of the partially defined medium used for the study of chromogenesis in Bacteroides

\section{Solution $A$}

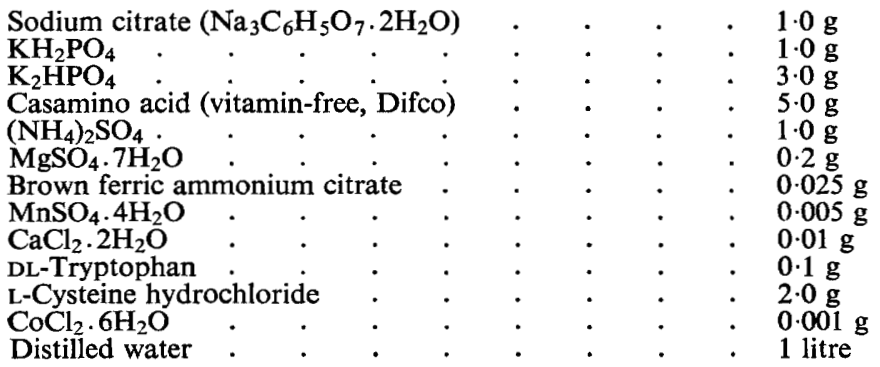

\section{Solution $B$}

p-Aminobenzoic acid
Pyridoxine :
Biotin
Riboflavin :
Cyanocobalamin :
Nicotinamide :
Adenine : :
Uracil :
Thymine :
Cytosine :
Guanine :
Solution A:

$\begin{array}{ll}0.1 \mathrm{ml} & (0.1 \mathrm{mg}) \\ 0.1 \mathrm{ml} & (0.1 \mathrm{mg}) \\ 1.0 \mathrm{ml} & (0.001 \mathrm{mg}) \\ 0.1 \mathrm{ml} & (0.1 \mathrm{mg}) \\ 0.1 \mathrm{ml} & (0.0001 \mathrm{mg}) \\ 0.1 \mathrm{ml} & (0.1 \mathrm{mg}) \\ 1.0 \mathrm{ml} & (1.0 \mathrm{mg}) \\ 1.0 \mathrm{ml} & (1.0 \mathrm{mg}) \\ 1.0 \mathrm{ml} & (1.0 \mathrm{mg}) \\ 1.0 \mathrm{ml} & (1.0 \mathrm{mg}) \\ 1.0 \mathrm{ml} & (1.0 \mathrm{mg})\end{array}$

For the complete medium solution $\mathbf{B}$ was freshly prepared and sterilised by autoclaving. After cooling, glucose was added to give a final concentration of 0.5 per cent. $w / v$ and ferrous sulphate to give a final concentration of 0.5 per cent. $\mathrm{w} / \mathrm{v}$. The reaction was adjusted to pH 7.2.

Depending on requirements vitamin $K_{1} 0.05$ per cent. w/v, haemin 0.001 per cent. w/v and serum $4 \mathrm{ml}$ per cent. $\mathrm{v} / \mathrm{v}$ were added immediately before use.

Extraction and purification of the pigment. The bacteria were separated from the pigment by centrifugation at 3000 r.p.m. for $20 \mathrm{~min}$., the supernatant being removed and retained. The process was repeated three times to ensure that most of the bacteria had been removed. The pigment was in very fine suspension, and showed no tendency to sediment during the procedure.

The pigment was examined spectroscopically in its crude form, after dilution in distilled water to a concentration of $0 \cdot 1$ per cent. $(\mathrm{v} / \mathrm{v})$. At that stage the pigment was suspended in the partially defined medium.

In an attempt to achieve a purer preparation a fresh suspension was fractionated by gel filtration through a Sephadex G-200 column (Pharmacia, G.B. Ltd, West Ealing, London), the fraction containing pigment being submitted for spectroscopy. Unfortunately, the pigment faded rapidly during the process of fractionation.

The third method used was the most satisfactory. After separation of the bacteria from the pigment by centrifugation, the supernatant was placed in a boiling waterbath for $1 \mathrm{hr}$ 
The pigment coagulated during this time, and was harvested easily by centrifugation at 3000 r.p.m. for $10 \mathrm{~min}$. After this, it was washed six times in sterile distilled water to remove all traces of the medium.

Spectroscopy showed an absorption peak at $250 \mathrm{~nm}$. This behaviour was not consistent with that of a haem pigment.

\section{RESULTS}

\section{Chromogenesis in undefined media}

The original observations were made with Robertson's medium for the cultivation of Bacteroides fragilis. The organism had been isolated from an appendix abscess, and was in mixed culture with Escherichia coli. A rusty nail had been added to the medium as a reducing agent. The culture was examined after overnight incubation and the medium was found to have turned ink-black. The procedure was repeated using a further six fresh isolates of $B$. fragilis. All were in mixed culture with either E. coli or Staphylococcus aureus, and all showed the same phenomenon. In pure culture, no discoloration of the medium was observed with any of the cultures, neither could the phenomenon be reproduced with combinations of E. coli or Staph. aureus with bacterial species other than those of the genus Bacteroides. All six strains of $B$. fragilis produced jet-black colonies on blood agar when initially isolated in mixed culture, but yielded colourless colonies in pure culture on the same medium.

The work was continued with the Bacteroides cultures obtained from the National Collection of Type Cultures. Chromogenesis with these cultures was most consistent in modified Starkey's medium. B. necrophorus rapidly produced total blackening of the medium, but $B$. fragilis failed to produce pigment without the addition of 0.001 per cent. (w/v) haemin. No growth of $B$. melaninogenicus was obtained in modified Starkey's medium.

\section{Chromogenesis in partially defined media}

B. necrophorus produced pigment readily and had three requirements for pigmentation, a source of iron (ferrous sulphate), cysteine and vitamin $K_{1}$. $B$. fragilis did not produce pigment as readily or as rapidly as $B$. necrophorus. It required haemin in addition to the three factors detailed above. Pigment production by this species proved to be erratic in the defined medium.

The role of haemin in pigmentation by $B$. melaninogenicus was difficult to evaluate as $B$. melaninogenicus requires haemin as a nutritional factor. However, the organism was able to dispense with vitamin $\mathrm{K}_{1}$ as a factor in chromogenesis. It required only the addition of cysteine and ferrous sulphate to produce a black pigment in the defined medium.

\section{The characteristics of the pigment}

Microscopy of the cultures showed that the pigment was extracellular. In fluid cultures it appeared as a dense ink-black suspension. On blood agar, colonies of $B$. melaninogenicum were haemolytic and jet black, the intensity of the colour increasing as the medium proceeded towards total haemolysis. 
After overnight exposure to the atmosphere, the colonies lost their shiny jet-black appearance, and turned brown. A similar phenomenon was noted in the case of pigment harvested from fluid cultures, which changed from a sooty black to a light yellow-brown colour.

A sample of the pigment, suspended in distilled water, was tested for solubility in various solvents. It was insoluble in chloroform, ether, alcohol and acetone. Initially, it appeared that it dissolved slowly in sodium hydroxide, but this impression was false. In fact the pigment merely decolorised on exposure to air, and was insoluble in sodium hydroxide.

Spectroscopy showed an absorption peak at $250 \mathrm{~nm}$.

\section{The identity of the pigment}

On chemical analysis, pigment from all four sources proved to have the characteristics of colloidal ferrous sulphide. Thus the observed colour change is explained, as ferrous sulphide readily oxidises to brown ferric sulphide.

\section{Discussion}

Both MacLennan (1951) and Sonnenwirth (1960) were of the opinion that the genus Bacteroides should be divided into three groups, the first being representative of pleomorphic strains, and typified by $B$. necrophorus, the second representing non-pleomorphic strains and typified by $B$. fragilis, and the third a miscellaneous group, of which $B$. melaninogenicus is a member.

Three strains, one representing each group, were employed in the present study. Each required a different set of factors for chromogenesis. These findings are summarised in table II and serve to reaffirm the distinction between the groups.

The taxonomy of the genus Bacteroides is confused: a number of illcharacterised and rarely isolated species have been described. Of all the members of the group, it is perhaps $B$. melaninogenicus that poses most problems.

There is an apparent difficulty in obtaining this organism in pure culture so that it has been studied only in mixed culture by some workers (Burdon, 1932; Lev, 1958; Gibbons and Macdonald, 1960). Thus the distinctive property of pigmentation has been the sole basis of identification of $B$. melaninogenicus, biochemical studies having been avoided, either on account of technical difficulties (Schwabacher et al., 1947) or because the organism could be maintained only in mixed culture.

Some investigators have recognised differences between strains of B. melaninogenicus. Weiss (1937) demonstrated biochemical and antigenic differences between his strains. Gibbons and Macdonald found variation in fermentative powers and also differences in nutritional requirements, there being two types, the first (or dependent type) requiring menadione (a vitamin-K analogue) and usually haemin, and the second (or independent type) requiring haemin but not menadione for growth. 
The present study offers an explanation for some of these findings. It has been shown that Bacteroides species other than B. melaninogenicus are capable of pigment production and that the chromogenic factors required correspond to the growth stimulatory factors of Gibbons et al. All these factors are present when Bacteroides species are isolated on blood agar in mixed culture. The blood agar is a source of haemin, and a vitamin K-like substance is produced by bacteria such as Escherichia coli and Staphylococcus aureus (Macdonald, Sutton and Knoll, 1954). If identification of B. melaninogenicus is attempted on the basis of chromogenesis alone, there may be confusion with other Bacteroides species that are chromogenic in mixed culture. In view of the reported antigenic and biochemical differences between strains of $B$. melaninogenicus it may be that this has occurred in the past.

TABLE II

The various chromogenic factors required by the three Bacteroides species studied

\begin{tabular}{|c|c|c|c|c|}
\hline \multirow{2}{*}{ Species } & \multicolumn{4}{|c|}{ Chromogenic factors } \\
\hline & Cysteine & Ferrous sulphate & Vitamin $\mathrm{K}_{1}$ & Haemin \\
\hline Bacteroides necrophorus & + & + & + & - \\
\hline Bacteroides fragilis & + & + & + & + \\
\hline Bacteroides melaninogenicus . & + & + & - & * \\
\hline
\end{tabular}

* It was not possible to assess the role of haemin in chromogenesis by B. melaninogenicus.

It might be anticipated that Bacteroides species producing pigmented colonies in mixed culture would yield colourless colonies in pure culture, in the absence of vitamin-K factor. This was found in the present study, and a similar phenomenon was described by Cohen (1932) and Lev (1958).

The first description of $B$. melaninogenicus was given by Oliver and Wherry (1921). They noted that the pigment was extracellular and that it was insoluble in chloroform, ether, alcohol and acetone but dissolved slowly in sodium hydroxide. On the basis of this behaviour, they identified it as melanin. This view held sway until Schwabacher et al. reviewed the nature of the pigment in 1947. They thought that the pigment was not melanin, but a breakdown product of haemoglobin, and devised an elaborate process for the extraction and purification of the pigment, involving the use of 90 per cent. phenol in the initial stages of treatment of the culture. The purified pigment was examined spectroscopically and was found to be identical with haemin.

The findings of Schwabacher et al. could be criticised on the grounds that they derived their material from a haemin-containing medium, and that they had found that haemin was a growth factor for $B$. melaninogenicus. They were alert to the problem of haemin contamination from the medium, and tried to circumvent it, but they did not attempt the separation of the bacterial cells 
from the pigment before treatment with phenol. The black pigment of B. melaninogenicus is extracellular, but it would appear that what Schwabacher et al. identified was not an extracellular pigment, but an intracellular one, as they stated that the haematin was united to a protein of the bacterial protoplasm, forming a parahaematin. It may be that the intracellular pigment identified as a parahaematin was a cytochrome. It is known that such pigments may occur in the obligate anaerobes (Postgate, 1956; White, Bryant and Caldwell, 1962).

In investigating the identity of the black pigment produced by $B$. melaninogenicus, Oliver and Wherry, using the crude methods of analysis available fifty years ago, came nearer to the truth than Schwabacher et al., who used sophisticated techniques. Formerly, melanin was believed to be a sulphur pigment derived from blood, so that the opinion of the original workers was not an unreasonable one. Neither is the name Bacteroides melaninogenicus entirely inappropriate, for the term melanin is derived from the Greek $\mu \epsilon^{\prime} \lambda \alpha s$, meaning black.

The function of the pigment remains obscure. However, since ferrous sulphide is a reducing agent, it is interesting to speculate that the pigment may facilitate the growth of the strictly anaerobic Bacteroides.

\section{SUMMARY}

Chromogenesis by three members of the genus Bacteroides was studied. The species investigated were: Bacteroides melaninogenicus, $B$. fragilis and $B$. necrophorus. All were capable of pigment production, but each species required a different set of factors for chromogenesis. All needed cysteine and ferrous sulphate. These factors sufficed for pigment production by B. melaninogenicus. Additional vitamin $\mathrm{K}_{1}$ was essential for pigment production by both $B$. necrophorus and $B$. fragilis, but the latter also required haemin.

Pigment production by $B$. melaninogenicus was studied in two media: 5 per cent. horse blood agar, and an agar-free partially defined medium. The pigment was harvested from these media, purified and subjected to chemical analysis. This pigment, previously thought to be haemin, proved to be colloidal ferrous sulphide.

The pigment produced by two other Bacteroides species, B. fragilis and $B$. necrophorus, examined in the same way also proved to be colloidal ferrous sulphide.

I should like to thank Professor Gy. Ivanovics for his advice and encouragement and Dr J. C. J. Ives for his interest in this work. The help of Dr N. McCorkindale, who performed the chemical analysis, was greatly appreciated and I am grateful to Dr J. McGeachie who carried out the spectroscopic examination.

\section{REFERENCES}

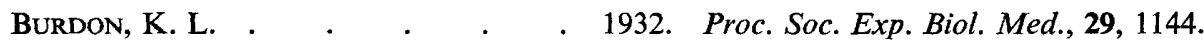

CoHEN, J. . . . . . . . . . 1932. Archs Surg., 24, 171.

Cowan, S. T., AND STEel, K. J. . . 1965. Manual for the identification of medical bacteria, London, p. 99. 
Gibbons, R. J., And Macdonald, J. B. . 1960. J. Bact., 80, 164.

LePPER, E., AND MARTIN, C. J. . . 1929. Br. J. Exp. Path., 10, 327.

LEV, M.

1958. Nature, Lond., 181, 203.

Macdonald, J. B., Sutton, R. M., And 1954. J. Infect. Dis., 95, 275.

KNOLL, M. L.

MacLennan, J. D. . . . . . 1951. Recent advances in bacteriology, 3rd ed., edited by J. H. Dible, London, p. 212.

Meynell, G. G., AND Meynell, Elinor 1965. Theory and practice in experimental bacteriology, London, p. 37.

Oliver, W. W., ANd Wherry, W. B. . 1921. J. Infect. Dis., 28, 341.

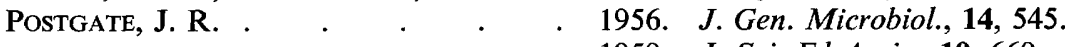

" $\quad$. . . . . . . 1959. J. Sci. Fd Agric., 10, 669.

ReEd, G. B., AND OrR, J. H. $\quad . \quad$. 1941. War Med., Chicago, 1, 493.

Schwabacher, Herta, Lucas, D. R., 1947. J. Gen. Microbiol., 1, 109. AND RIMINGTON, C.

Sonnenwirth, A. C. . . . $\quad$. 1960. Ph.D. Dissertation, St Louis, Washington University.

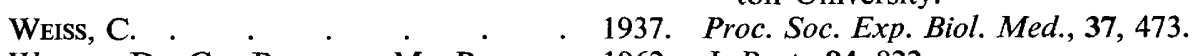

White, D. C., BRyant, M. P., AND 1962. J. Bact., 84, 822.

Caldwell, D. R. 